ArtefaCToS. Revista de estudios de la ciencia y la tecnología

eISSN: $1989-3612$

Vol. 9, No. 1 (2020), 2. ${ }^{\text {Época, 5-12 }}$

DOI: https://dx.doi.org/10.14201/art202091512

\title{
Presentación
}

\section{Nuevos enfoque en Ciencias Sociales y análisis de las Ciencias de lo Artificial}

Presentation

New Approaches in the Social Sciences and Analysis of the Sciences of the Artificial

\author{
Wenceslao J. GONZÁLEZ \\ Editor invitado/Guest editor \\ Centro de Investigación de Filosofía de la Ciencia y la Tecnología, \\ Universidad de A Coruña, España \\ wenceslao.gonzalez@udc.es
}

\section{Resumen}

Mediante el número monográfico "De las Ciencias Sociales a las Ciencias de lo Artificial: Nuevas perspectivas filosóficas”, se busca ampliar el radio de acción filosófico-metodológico habitual sobre la Ciencia. Así, el marco conceptual viene dado por dos factores principales: 1) propiciar un mayor reconocimiento de las Ciencias Sociales como campo de reflexión, pues habitualmente reciben mucha menos atención que las Ciencias de la Naturaleza; y 2) resaltar la necesidad de abordar cuestiones de las Ciencias de lo Artificial, debido a la creciente importancia de las Ciencias de Diseńo en el mundo contemporáneo, que contrasta con la escasa presencia que tienen en la tendencia dominante en Filosofía y Metodología actual de la Ciencia. Los contenidos del presente número monográfico inciden en varios aspectos: (i) el ofrecer novedades en términos horizontales o longitudinales, que lleva a considerar nuevos enfoques, especialmente en las disciplinas de Ciencias Sociales; y (ii) buscar novedades verticales o transversales, para tratar nuevos temas y profundizar en disciplinas que no han recibido atención suficiente, sobre todo en el campo de las Ciencias de Diseño. Dentro de ese marco se dan detalles sobre los artículos del presente volumen.

Palabras clave: novedad; planteamientos; estudio; sociedad; saberes; diseño. 


\begin{abstract}
Through the monographic issue "From the Social Sciences to the Sciences of the Artificial: New Philosophical Perspectives", the aim to broaden the usual philosophical-methodological scope of action on science. Thus, the conceptual framework is provided by two main factors: 1) to foster greater recognition of the social sciences as a field of reflection, since they usually receive much less attention than the natural sciences; and 2) to highlight the need to address issues of the sciences of the artificial, due to the growing importance of the design sciences in the contemporary world, which contrasts with the scarce presence they have in the dominant trend in contemporary philosophy and methodology of science. The contents of this monographic issue have a bearing on several aspects: (i) offering novelties in horizontal or longitudinal terms, which leads to the consideration of new approaches, especially in the disciplines of the social sciences; and (ii) looking for vertical or transversal novelties, to deal with new issues and to go deeper into disciplines that have not received sufficient attention, especially in the field of design sciences. It is within this framework that details are given of the articles in this volume.
\end{abstract}

Keywords: novelty; prospects; study; society; knowledge; design.

\title{
1. Marco conceptual de las nuevas perspectivas filosóficas
}

Surge este número monográfico del deseo de resaltar nuevas perspectivas filosóficas acerca de las Ciencias Sociales y las Ciencias de lo Artificial. Busca ampliar el radio de acción filosófico-metodológico habitual sobre la Ciencia. Por eso, los artículos abordan cuestiones desde ángulos distintos, en sintonía con el pluralismo metodológico, y tratan también cuestiones genuinamente nuevas en este campo temático. Se centra, en efecto, en dos relevantes grupos de Ciencias empíricas, como son las Ciencias Sociales y las Ciencias de lo Artificial. Constituyen el eje temático del volumen porque, hasta la fecha, no han recibido el mismo nivel de atención filosófico-metodológico que las Ciencias de la Naturaleza. Complementa así este número monográfico lo aportado hace unos ańos, cuando llamaba la atención sobre Ciencias que la Filosofía ha descuidado (neglected) y planteaba nuevos desafíos (González, 2013).

Sucede que, en las últimas dos décadas, algunas Ciencias Sociales - principalmente, la Economía y la Psicología - cuentan, sin duda, con publicaciones específicas (monografías, libros de edición, artículos de revista, etc.) y con trabajos en Congresos nacionales e internacionales organizados dentro del marco de la tendencia dominante en Filosofía y Metodología de la Ciencia ${ }^{1}$. Pero, gene-

\footnotetext{
${ }^{1}$ Se entiende por la tendencia dominante en Filosofía y Metodología de la Ciencia aquella que, cada cuatro años, se refleja en los Congresos de Logic, Methododology and Philosophy of Science and Technology (CLMPST), o en los encuentros bianuales de la European Philosophy of Science
} 
ralmente, están situadas en un segundo plano y con un número de aportaciones comparativamente menor, si se contrasta con las contribuciones relacionadas con el conjunto de las Ciencias de la Naturaleza y, de modo especial, si se trata de textos sobre Física o Biología.

Un ejemplo ilustrativo es el Programa europeo The Philosophy of Science in a European Perspective, financiado por la European Science Foundation, que duró de 2008 a 2013. Estaba organizado en cinco equipos, que fueron el soporte cada año a otros tantos Simposios, con una amplia participación de filósofos de la Ciencia europeos. Uno de los equipos estaba dedicado a la "Filosofía de la Cultura y de las Ciencias Sociales"2. Mientras tanto, de los otros cuatro equipos, dos estaban expresamente centrados en Ciencias de la Naturaleza: "Filosofía de las Ciencias de la Vida" y "Filosofía de las Ciencias Físicas", y los otros restantes, que giraban en torno a "Métodos formales" e "Historia de la Filosofía de la Ciencia", estaban también volcados habitualmente hacia las Ciencias de la Naturaleza.

Esto se reflejó en los cinco volúmenes que, publicados por la editorial Springer, dieron a luz a los trabajos que finalmente fueron aceptados para la publicación. Fueron los libros siguientes: The Present Situation in the Philosophy of Science (Stadler et al., 2010); Explanation, Prediction, and Confirmation (Dieks et al., 2011); Probabilities, Laws, and Structures (Dieks et al., 2012); New Challenges to Philosophy of Science (Andersen et al., 2013); y New Directions in the Philosophy of Science (Galavotti et al., 2014).

Junto a aportaciones directamente enfocadas hacia cuestiones filosófico-metodológicas relacionadas con la actividad social (económica, psicológica, jurídica, etc.), parte de los trabajos sobre Filosofía de las Ciencias Sociales eran, en rigor, estudios comparativos con Filosofía de las Ciencias de la Naturaleza. Pero, a su vez, un rasgo relevante del Programa europeo era la clara conciencia de la existencia de Ciencias - algunas de ellas nuevas - que, hasta ahora, han sido ignoradas por la literatura filosófica especializada. Por eso, uno de los años del Programa europeo - el cuarto - estuvo centrado en reflexionar sobre esas disciplinas.

Association (EPSA) o de la Philosophy of Science Association (PSA) de Estados Unidos y en sus correspondientes publicaciones. También se refleja en los Congresos anuales de sociedades como la British Society for the Philosophy of Science o de otras naciones y en las revistas relacionadas con esas sociedades.

${ }^{2}$ En el caso de Philosophy of the Cultural and Social Sciences, donde Wenceslao J. González era el líder del equipo - el C-y Amparo Gómez era la colíder, los Workshops organizados fueron los siguientes: "The Present Situation of the Philosophy of the Cultural and Social Sciences: The 'Naturalist Turn', the 'Social Turn', and the Discussion on Scientific Realism” (Universidad de Viena, 2008); "Explanation, Prediction, and Confirmation in the Social Sciences: Realm and Limits" (Universidad de Ámsterdam, 2009); “The Debate on Mathematical Modeling in the Social Sciences" (Universidad de A Coruña, Campus de Ferrol, 2010); "The Sciences of the Artificial vs. The Cultural and Social Sciences" (National School of Political Studies and Public Administration, Bucarest, 2011); y "The Social Concern on Science: Social Constructivism and Realism on the Social Sciences" (Universidad de Bolonia, 2012). 
Mientras tanto, es ciertamente muy infrecuente el encontrar estudios filosófico-metodológicos sobre las Ciencias de lo Artificial, en general, y las Ciencias de Diseño, en particular ${ }^{3}$. Es un campo de estudio configurado primordialmente por Herbert A. Simon, cuyo libro The Sciences of the Artificial (1996) sigue siendo fuente de ideas. Acontece, además, que es precisamente en el terreno de las Ciencias de lo Artificial donde se están dando muchas novedades, que son de muy diverso tipo — semánticas, epistemológicas, metodológicas, ontológicas, axiológicas y éticas - y atañen de lleno a la Ciencia en el mundo actual. Este es precisamente el caso de las Ciencias de Internet, que pueden ser abordadas directamente desde las Ciencias de Diseño (González, 2018a; González y Arrojo, 2019).

Sobre la Filosofía de las Ciencias de lo Artificial, este número monográfico desea resaltar la tarea de la Filosofía acerca de las Ciencias de Diseño, entendidas sobre la base de las aportaciones realizadas por Simon (1996), pero matizadas a través de contribuciones posteriores. Se busca suplir aspectos que no tuvo en cuenta, tales como la racionalidad de fines o evaluativa —en lugar de la primacía completa de la racionalidad instrumental o de medios-, la relevancia de la actividad humana frente a la mera conducta, el papel de la historicidad como clave del cambio - más versátil y rica en matices que la evolución- etc. (González 2007 y 2012b). Se trata, además, de analizar disciplinas de las que, de facto, no fueron objeto de su atención: las Ciencias de la Comunicación, las Ciencias de la Documentación y la Ciencia de la Educación.

Conviene resaltar que, debido al impacto de Internet, estamos en un contexto histórico-social nuevo, donde hay una expansión por la dimensión de lo artificial, mediante la interacción entre creatividad científica e innovación tecnológica. Esto propicia la configuración de nuevas disciplinas científicas — sobre todo, las relacionadas con la Red de redes- y novedosas formas de interrelación entre las ya existentes (interdisciplinariedad, transdisciplinariedad, intersección disciplinar, etc.). La importancia de esta etapa histórica es inequívoca. Más aún, cabe pensar que ya no estamos en la Historia Contemporánea, sino en una nueva distinta y posterior, relacionada con la Sociedad del Conocimiento: la Hiperhistoria (Floridi, 2014, 1-24, 167-168 y 185).

\footnotetext{
${ }^{3}$ Esta fue la razón de organizar en Bucarest, dentro del Programa europeo, el Simposio sobre "The Sciences of the Artificial vs. The Cultural and Social Sciences", celebrado del 15 al 16 de septiembre de 2011. El título del Workshop quería resaltar que las Ciencias de lo Artificial no son lo mismo que las Ciencias de la Cultura o que las Ciencias Sociales, si bien hay Ciencias que puede ser duales (esto es, Ciencias de lo Artificial y Ciencias Sociales). Es el caso, a mi juicio, de la Economía, las Ciencias de la Documentación y las Ciencias de la Comunicación (González, 2008y 2012a).
} 


\section{Contenidos del presente número monográfico}

Dentro del marco conceptual señalado, el presente número monográfico ofrece artículos en los dos campos señalados: la Filosofía de las Ciencias Sociales y la Filosofía de las Ciencias de lo Artificial, que incluye también textos que combinan ambos aspectos. Al primer grupo pertenecen los enfoques sobre Psicología y causalidad, desde dos perspectivas distintas, como son la intervencionista de James Woodward ${ }^{4}$, que prescinde de componentes antropológicos 5 , y la basada en una concepción de la causalidad a partir de la agencia, donde se combina causalidad e indeterminismo, que propone Rafaela García Elskamp. Después están los trabajos sobre la dimensión social de la Tecnología, de Brian Balmer, que analiza el control de las armas biológicas y químicas en el contexto de las relaciones entre Ciencia, Tecnología y Sociedad, y la Filosofía de la innovación, vista en conexión con los valores sociales en las empresas, de Javier Echeverría.

Dos de los artículos combinan de manera clara la Filosofía de las Ciencias Sociales y la Filosofía de las Ciencias de lo Artificial. Se trata del estudio de la dimensión social de Internet, realizado a partir del análisis filosófico-metodológico de la complejidad, de Wenceslao J. González, y la propuesta de las Ciencias de la Comunicación desde el realismo científico, a tenor del problema de la complejidad y las noticias falsas (fake news), de María José Arrojo. Se abordan así dos cuestiones de gran actualidad e indudable relevancia, no solo desde un punto de vista intelectual sino también desde la perspectiva de la vida social en un mundo globalizado.

Hay dos artículos que inciden más en las Ciencias de Diseño, dentro del marco de las Ciencias de lo Artificial. Es el caso de la consideración de los límites de las Ciencias de la Documentación, donde los límites se analizan en los tres pasos sucesivos de objetivos, procesos y resultados, que lleva a cabo Antonio Bereijo, y la indagación sobre la Ciencia de la Educación desde un punto de vista metodológico, que lleva a atender las diversas opciones metodológicas de índole disciplinar (la específica, la interdisciplinar, la multidisciplinar, la transdisciplinar, ...), de Ana María Alonso.

Como las Ciencias de Diseño constituyen un grupo de disciplinas científicas que, ciertamente, no ha recibido suficiente atención por parte de los filósofos que reflexionan sobre la actividad científica, puede que llamen más la atención estos estudios sobre las Ciencias de Internet, las Ciencias de la Comunicación, las Ciencias de la Documentación y las Ciencias de la Educación. Son temas que se trabajan habitualmente en el Centro de Investigación de la Filosofía de la Ciencia y la Tecnología (CIFCYT) de la Universidad de A Coruña, constituido

\footnotetext{
${ }^{4}$ Su postura sobre la causalidad es desarrollada en detalle en Woodward (2003/2005).

${ }^{5}$ Este rasgo de ausencia de componentes antropológicos en esta posición intervencionista es resaltado en Gonzalez (2018c).
} 
en el año 2017, que es ahora la entidad organizadora de las Jornadas de Filosofía y Metodología actual de la Ciencia, que han cumplido recientemente los 25 años.

Uno de los objetivos del Centro de Investigación es llamar la atención sobre la necesidad de ampliar el radio de acción de la Filosofía de la Ciencia, para no verse reducida a las disciplinas más tradicionales o consolidadas, y ocuparse así de las diversas expresiones científicas, en lugar de las más conocidas y frecuentemente analizadas. Esto supone reconocer la creciente influencia de las Ciencias de Diseño en el mundo actual y abrirse a temas nuevos. También adentrarse en las Ciencias de lo Artificial, especialmente en el caso de las Ciencias de Internet, supone reconocer la importancia de saber integrar la perspectiva científica, la faceta tecnológica y la dimensión social, como ya se anticipó en un trabajo anterior (González, 2018b).

Varios de contenidos de este número monográfico tienen su origen en trabajos inicialmente presentados en las Jornadas de Filosofía y Metodología actual de la Ciencia $^{6}$, organizadas por la Universidad de A Coruña con el respaldo de la Sociedad de Lógica, Metodología y Filosofía de la Ciencia en España. Es el caso de "Teoría normativa y Psicología descriptiva en la comprensión del razonamiento causal: Papel de las intervenciones y la invarianza", ponencia presentada por James Woodward el 13 de marzo de 2014. Se publica aquí según la traducción de Amanda Guillán para la edición XIX de las Jornadas.

También se publica ahora la versión original de la ponencia de Brian Balmer: "La dimensión social de la Tecnología: El control de las armas biológicas y químicas". La presentó el 16 de marzo 2012 en las Jornadas sobre Tecnología, valores y Ética, la edición XVII de las Jornadas. La traducción ha sido realizada asimismo por Amanda Guillán. Mientras tanto, el texto que ahora se publica de "Causalidad e indeterminismo en la acción humana. Reflexiones sobre causas y razones”, de Rafaela García Elskamp, es una versión ampliada y actualizada del trabajo inicial. Lo presentó en las Jornadas sobre Libertad y determinismo: Ciencias Sociales y Ciencias de la Naturaleza, que fueron la edición XIII de las Jornadas. También el trabajo de Antonio Bereijo, titulado "Los límites de las Ciencias de la Documentación: Límites de objetivos, procesos y resultados", es una versión revisada respecto de lo que presentó en las Jornadas sobre Limites de la Ciencia, que fueron las XVIII Jornadas, las últimas a las que puedo asistir.

Tiene origen distinto el artículo de Javier Echeverría, dedicado a la "Filosofía de la innovación y valores sociales en las empresas". Fue inicialmente una ponencia presentada en Encuentro sobre Innovación social e innovación tecnológica, organizado por Centro de Investigación de la Filosofía de la Ciencia y la Tecnología

\footnotetext{
${ }^{6}$ Los trabajos que se publican a partir de las Jornadas se reúnen en la Colección Gallaecia: Estudios de Filosofía y Metodología actual de la Ciencia. La información a este respecto se encuentra disponible en https://cifcyt.udc.es/coleccion-gallaecia/ (acceso 3.4.2020). Ahí están los datos de los títulos ya publicados, tanto de libros como de números monográficos de revista.
} 
(CIFCYT) y celebrado el 24 de octubre de 2018 en la Universidad de A Coruña, Campus de Ferrol. El texto se ofrece ahora en una versión revisada y actualizada para este número monográfico de Artefactos.

Orientados directamente a este volumen hay tres trabajos, que inciden en las Ciencias de Diseño desde distintos ángulos. Se trata de los artículos "La dimensión social de Internet: Análisis filosófico-metodológico desde la complejidad", de Wenceslao J. González; "Las Ciencias de la Comunicación desde el realismo científico: El problema de la complejidad y las noticias falsas (fake news)", de María José Arrojo; y "La Ciencia de la Educación ante las diversas opciones metodológicas de índole disciplinar", de Ana María Alonso.

Finalmente, deseo agradecer la amable invitación de Obdulia Torres, coeditora de la revista a preparar un número monográfico de Artefactos sobre las nuevas perspectivas filosóficas en Ciencias Sociales y en Ciencias de lo Artificial. Esa novedad es dual: por un lado, está la novedad horizontal o longitudinal, que consiste en expandir el territorio, como sucede aquí en casos como las cuestiones relacionadas con la Psicología y la causalidad; y, por otro lado, está la novedad vertical o transversal, que lleva a plantear temas radicalmente nuevos para la Filosofía y Metodología actual de la Ciencia, tales como las Ciencias de Internet o las Ciencias de la Documentación, o se ocupa de asuntos que han estado habitualmente desatendidos por falta de un enfoque adecuado para abordarlos, tales como las Ciencias de la Comunicación o las Ciencias de la Educación.

\section{Referencias bibliográficas}

Andersen, Hanne, Dieks, Dennis, Gonzalez, Wenceslao J., Uebel, Thomas y Wheeler, Gregory (eds.) (2013). New Challenges to Philosophy of Science. Dordrecht: Springer.

Dieks, Dennis, Gonzalez, Wenceslao J., Hartman, Stephan, Uebel, Thomas y Weber, Marcel (eds.) (2011). Explanation, Prediction, and Confirmation. Dordrecht: Springer.

Dieks, Dennis, Gonzalez, Wenceslao J., Hartman, Stephan, Stöltzner, Michael y Weber, Marcel (eds.) (2012). Probabilities, Laws, and Structures. Dordrecht: Springer.

Floridi, Luciano (2014). The Fourth Revolution - How the Infosphere is Reshaping Human Reality. Oxford: Oxford University Press.

Galavotti, Maria Carla, Dieks, Dennis, Gonzalez, Wenceslao J., Hartman, Stephan, Uebel, Thomas y Weber, Marcel (eds.) (2014). New Directions in the Philosophy of Science. Dordrecht: Springer.

González, Wenceslao J. (2007). Configuración de las Ciencias de Diseño como Ciencias de lo Artificial: Papel de la Inteligencia Artificial y de la racionalidad limitada. En González, Wenceslao J. (ed.), Las Ciencias de Diseño: Racionalidad limitada, predicción y prescripción (pp. 41-69). A Coruña: Netbiblo. 
Gonzalez, Wenceslao J. (2008). Rationality and Prediction in the Sciences of the Artificial: Economics as a Design Science. En Galavotti, Maria Carla, Scazzieri, Roberto y Suppes, Patrick (eds.), Reasoning, Rationality, and Probability (pp. 165186). Stanford: CSLI Publications.

González, Wenceslao J. (2012a). Las Ciencias de Diseño en cuanto Ciencias de la Complejidad: Análisis de la Economía, Documentación y Comunicación. En González, Wenceslao J. (ed.), Las Ciencias de la Complejidad: Vertiente dinámica de las Ciencias de Diseño y sobriedad de factores (pp. 7-30). A Coruña: Netbiblo.

González, Wenceslao J. (2012b). La vertiente dinámica de las Ciencias de la Complejidad. Repercusión de la historicidad para la predicción científica en las Ciencias de Diseño. En González, Wenceslao J. (ed.), Las Ciencias de la Complejidad: Vertiente dinámica de las Ciencias de Diseño y sobriedad de factores (pp. 73-106). A Coruña: Netbiblo.

Gonzalez, Wenceslao J. (2013). From the Sciences that Philosophy has 'Neglected' to the New Challenges. En Andersen, Hanne, Dieks, Dennis, Gonzalez, Wenceslao J., Uebel, Thomas y Wheeler, Gregory (eds.), New Challenges to Philosophy of Science (pp. 1-6). Dordrecht: Springer.

González, Wenceslao J. (2018a). Complejidad dinámica en Internet como plataforma de información y comunicación: Análisis filosófico desde la perspectiva de Ciencias de Diseño y el papel de la predicción. Informação e Sociedade: Estudos, 28(1), 155-168.

González, Wenceslao J. (2018b). Internet en su vertiente científica: Predicción y prescripción ante la complejidad. Artefactos: Revista de Estudios sobre Ciencia y Tecnologia, 7(2), 2a época, 75-97. https://dx.doi.org/10.14201/art2018717597

Gonzalez, Wenceslao J. (2018c). Configuration of Causality and Philosophy of Psychology: An Analysis of Causality as Intervention and its Repercussion for Psychology. En Gonzalez, Wenceslao J. (ed.), Philosophy of Psychology: Causality and Psychological Subject. New Reflections on James Woodward's Contribution (pp. 21-70). Boston/Berlín: De Gruyter.

Gonzalez, Wenceslao J. y Arrojo, María J. (2019). Complexity in the Sciences of the Internet and its Relation to Communication Sciences. Empedocles: European Journal for the Philosophy of Communication, 10(1), 15-33. https://doi.org/10.1386/ ejpc.10.1.15_1

Simon, Herbert A. (1996). The Sciences of the Artificial, $3^{\text {a }}$ ed. Cambridge, MA: The MIT Press (1a ed., 1969; 2a 1981).

Stadler, Friedrich, Dieks, Dennis, Gonzalez, Wenceslao J., Hartman, Stephan, Uebel, Thomas y Weber, Marcel (eds.) (2010). The Present Situation in the Philosophy of Science. Dordrecht: Springer.

Woodward, James (2003). Making Things Happen: A Theory of Causal Explanation. N. York: Oxford University Press (edición en paperback, septiembre de 2005). 